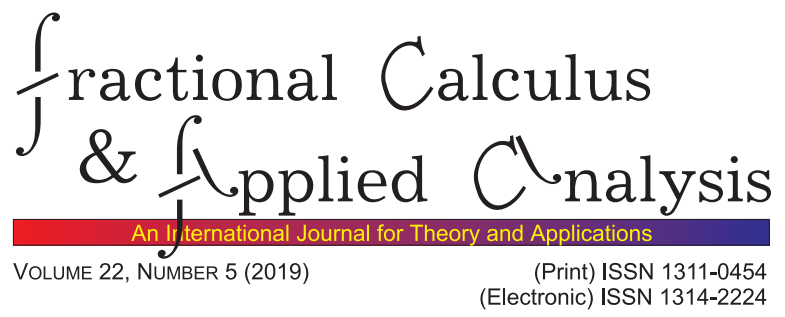

RESEARCH PAPER

\title{
EMBEDDINGS OF WEIGHTED GENERALIZED MORREY SPACES INTO LEBESGUE SPACES ON FRACTAL SETS
}

\author{
Natasha Samko
}

\begin{abstract}
We study embeddings of weighted local and consequently global generalized Morrey spaces defined on a quasi-metric measure set $(X, d, \mu)$ of general nature which may be unbounded, into Lebesgue spaces $L^{s}(X), 1 \leq$ $s \leq p<\infty$. The main motivation for obtaining such an embedding is to have an embedding of non-separable Morrey space into a separable space.

In the general setting of quasi-metric measure spaces and arbitrary weights we give a sufficient condition for such an embedding. In the case of radial weights related to the center of local Morrey space, we obtain an effective sufficient condition in terms of (fractional in general) upper Ahlfors dimensions of the set $X$. In the case of radial weights we also obtain necessary conditions for such embeddings of local and global Morrey spaces, with the use of (fractional in general) lower and upper Ahlfors dimensions. In the case of power-logarithmic-type weights we obtain a criterion for such embeddings when these dimensions coincide.
\end{abstract}

$M S C$ 2010: Primary 46E30; Secondary 43A85, 28A80

Key Words and Phrases: weighted Morrey spaces; quasi-metric measure spaces; fractal sets; upper and lower fractional dimensions; embedding

\section{Introduction}

Last decades there increases an interest to the study of function spaces on sets of complicated structure, often in the setting of quasi-metric measure spaces, in particular, on fractal sets, as well as to operator theory in

(C) 2019 Diogenes Co., Sofia

pp. 1203-1224, DOI: 10.1515/fca-2019-0064

DE GRUYTER 
such spaces. We refer, for instance, to the books [15], [26] and [28] and papers [3], 44, [5], 6], 9], [12], [16], 20], 22] and references therein. In this paper we consider weighted generalized Morrey spaces on underlying quasi-metric measure sets. Morrey spaces in such a setting were studied, for instance, in [8, 13], 14], [16], [17, 18], 19], 24], [27].

We study embeddings of weighted local generalized Morrey spaces $L_{\left\{x_{0}\right\}}^{p, \varphi}(X, w), 1<p<\infty$, defined on unbounded quasi-metric measure sets $(X, d, \mu)$ of general nature, into Lebesgue space $L^{s}(X), 1 \leq s \leq p$. This immediately implies the same embedding for corresponding global weighted generalized Morrey spaces.

The main motivation of such a study is to obtain an embedding of the non-separable space $L_{\left\{x_{0}\right\}}^{p, \varphi}(X, w)$ into a separable space. Such an embedding helps to derive some relations between operators acting in Morreytype spaces without using their continuation from "nice" functions in these spaces when these relations hold in a larger space to which the non-separable Morrey space is embedded. For instance this idea was used in [21] in the study of Fredholm properties of singular integral operators in non-separable generalized Hölder spaces.

In the Euclidean setting, embeddings between weighted global classical Morrey spaces were studied in [10], where the case of Lebesgue spaces is not admitted. We also refer to the papers [1] and [23, where was shown that the non-weighted local Morrey space is closely embedded between two weighted Lebesgue spaces with "small gap" between weights.

In the general setting of quasi-metric measure spaces and arbitrary weights we give a sufficient condition for the embedding

$$
L_{\left\{x_{0}\right\}}^{p, \varphi}(X, w) \hookrightarrow L^{s}(X), \quad 1 \leq s \leq p
$$

of local generalized Morrey spaces, related to the point $x_{0} \in X$. Hence the same embedding holds for the corresponding global space. In this general condition we do not suppose that $(X, d, \mu)$ satisfies either upper Ahlfors condition (growth condition) or lower Ahlfors condition (doubling condition).

In the case of "radial" weights $w$ depending on the distance $d\left(x, x_{0}\right)$ we give sufficient conditions in a more effective form in terms of a certain one-dimensional integral condition imposed on $\frac{\varphi(r)^{\frac{1}{p}}}{w(r)}$ if we assume that the growth condition holds. We also obtain necessary conditions for studied embeddings of both local and global Morrey spaces. While the sufficient conditions involve only the upper fractional Ahlfors dimension, the necessary conditions involve both upper and lower dimensions. In the case of power-logarithmic-type weights we obtain coinciding necessary and sufficient conditions on the weight $w$ when these dimensions coincide with each 
other. We also provide application to the case of radial weights $w\left[d\left(x, x_{0}\right)\right]$ of power-logarithmic-type with different exponents for small and big values of $d\left(x, x_{0}\right)$ (assuming that $X$ is unbounded in this case) and $\varphi(r)$ is a power-type function, also with different exponents at the origin and infinity. In the choice of these examples we follow the paper [10. In this case we arrive at necessary and sufficient conditions for embeddings of both local and global Morrey spaces, in terms of numerical inequalities for the exponents of the weight $w$ and function $\varphi$, when the lower and upper Ahlfors dimensions coincide with each other.

The paper is organized as follows. In Section 2 we provide necessary information on quasi-metric measure spaces $(X, d, \mu)$ including information on lower and upper, fractional in general, Ahlfors dimensions and some technical lemmas related to these dimensions. The definition of generalized Morrey spaces on $(X, d, \mu)$ may be also found in this section. Section 3 contains the main results. In Subsection 3.1 we deal with arbitrary weights and provide a general condition where neither growth nor doubling condition is imposed on $(X, d, \mu)$. In Subsection 3.2 we treat the case of radial weights, where we obtain more efficient conditions directly expressed in terms of the upper Ahlfors dimension, with a special attention for the case of powerlogarithmic weights. In Subsection 3.3, we study necessary conditions for the embeddings. Finally, in Subsection 3.4 we illustrate the obtained results for the case where plane fractal curves are chosen as an example of quasi-metric measure space.

Everywhere in the sequel $c, C, C_{1}, C_{2}$ etc. denote positive absolute constants which may have different values in the same lines. The equivalence $f \cong g$ for non-negative functions $f$ and $g$ is used in the sense that $c_{1} f(x) \leq g(x) \leq c_{2} f(x)$, where $c_{1}>0$ and $c_{2}>0$ do not depend on $x$.

\section{Preliminaries}

2.1. Quasi-metric measure spaces $(X, d, \mu)$. Let $X$ be a set equipped with a positive measure $\mu$ and a quasi-distance $d$ :

$$
d(x, y) \leq k[d(x, z)+d(y, z)], \quad k \geq 1,
$$

$d(x, y)=0 \Longleftrightarrow x=y, \quad d(x, y)=d(y, x)$.

The triplet $(X, d, \mu)$ is called quasi-metric measure space. For quasimetric measure spaces and for function spaces on them we refer, for instance, to [7] and [1].

We use the standard notation $B(x, r):=\{y \in X: d(x, y)<r\}$.

Everywhere in the sequel $\mu$ is a positive measure on the $\sigma$-algebra of subsets of $X$ which contains balls $B(x, r)$. We say that $(X, d, \mu) \in \mathfrak{X}$ if

(1) all balls $B(x, r)$ are open sets of finite measure, $x \in X, 0<r<\ell$, where $\ell=\operatorname{diam} X, 0<\ell \leq \infty$; 
(2) the spheres $S(x, r):=\{y \in X: d(y, x)=r\}$ have zero measure for all $x$ and $r$.

In some statements in the sequel we will use the following assumption on the growth of measures of balls: there exists a constant $m>1$ such that

$$
\mu B(x, 2 r) \geq m \mu B(x, r), \quad 0<r<\ell .
$$

Definition 2.1. Let $\ell<\infty$. The triplet $(X, d, \mu)$ is said to satisfy the lower Ahlfors condition if there exist the constant $C>0$ and an exponent $\tau_{0}>0$ such that

$$
\mu B(x, r) \geq c r^{\tau_{0}}, 0<r<\ell, x \in X .
$$

In the case $\ell=\infty$, the condition of the type (2.3), i.e.

$$
\mu B(x, r) \geq c r^{\tau_{0}}, 0<r<1, x \in X
$$

with some $C>0$ and $\tau_{0}>0$, will be referred to as local lower Ahlfors condition and the condition

$$
\mu B(x, r) \geq c r^{\tau_{\infty}}, 1<r<\infty, x \in X .
$$

will be called global lower Ahlfors condition.

In the case where one of the inequalities (2.3)-(2.5) holds only at a single point $x=x_{0} \in X$, we say that $(X, d, \mu)$ has the corresponding lower Ahlfors property at the point $x_{0}$.

Definition 2.2. Let $\ell<\infty$. The triplet $(X, d, \mu)$ is said to satisfy the upper Ahlfors condition if there exist the constant $C>0$ and an exponent $\sigma_{0}>0$ such that

$$
\mu B(x, r) \leq c r^{\sigma_{0}}, 0<r<\ell, x \in X .
$$

In the case $\ell=\infty$, the condition of the type (2.6), i.e.

$$
\mu B(x, r) \leq c r^{\sigma_{0}}, 0<r<1, x \in X
$$

with some $C>0$ and $\sigma_{0}>0$, will be referred to as local upper Ahlfors condition and the condition

$$
\mu B(x, r) \leq c r^{\sigma_{\infty}}, 1<r<\infty, x \in X .
$$

will be called global upper Ahlfors condition.

In the case where one of the inequalities (2.6)-(2.8) holds only at a single point $x=x_{0} \in X$, we say that $(X, d, \mu)$ has the corresponding upper Ahlfors property at the point $x_{0}$.

The upper Ahlfors conditions are also known as growth conditions. It is known that the doubling property of measure

$$
\mu B(x, r): \mu B(x, 2 r) \leq C \mu B(x, r), \quad 0<r<\ell \leq \infty
$$


implies the local lower Ahlfors conditions (2.3) and (2.4). Under the doubling condition the quasi-metric measure space $(X, d, \mu)$ is usually called space of homogeneous type.

Clearly, the inequalities (2.3)-(2.5) and (2.6)-(2.8) themselves do not determine uniquely the exponents $\tau_{0}, \tau_{\infty}, \sigma_{0}$ and $\sigma_{\infty}$. We say that these exponents are precise if they cannot be improved. For instance, the exponent $\sigma_{0}$ is precise if (2.6) holds with this $\sigma_{0}$ but does not hold if $\sigma_{0}$ is replaced by $\sigma_{0}+\varepsilon$. When the exponents $\tau_{0}, \tau_{\infty}, \sigma_{0}$ and $\sigma_{\infty}$ are precise, they are referred to as lower and upper dimensions of $(X, d, \mu)$, local and global, respectively. These dimensions are fractional in general.

In the case where $X$ is bounded, we say that the quasi-metric measure space $(X, d, \mu)$ is regular if $\sigma_{0}=\tau_{0}$. In the case of unbounded set we say that $(X, d, \mu)$ is locally regular if $\sigma_{0}=\tau_{0}$, and globally regular if $\sigma_{\infty}=\tau_{\infty}$. Finally, we say that the triplet $(X, d, \mu)$ with an unbounded set $X$ is regular if it is both locally and globally regular and $\tau_{0}=\sigma_{0}=\tau_{\infty}=\sigma_{\infty}$.

2.2. Technical lemmas. Recall that a non-negative function $\varphi$ on an interval $(a, b) \in \mathbb{R}$, is called almost increasing (almost decreasing), if there exists a constant $C(\geq 1)$ such that $\varphi(x) \leq C \varphi(y)$ for all $a<x \leq y<b$ ( $b>x \geq y>a$, respectively). Equivalently, a function $\varphi$ is almost increasing (almost decreasing), if it is equivalent to an increasing (decreasing), respectively, function $\psi$, i.e. $\varphi(x) \cong \psi(x)$. For a function $\varphi$ non-negative on an interval $(a, b)$, almost increasing (decreasing), the constants

$$
C_{\varphi}:=C_{\varphi}(a, b)=\sup _{a<t<T<b} \frac{\varphi(t)}{\varphi(T)} \text { and } c_{\varphi}:=c_{\varphi}(a, b)=\sup _{a<t<T<b} \frac{\varphi(T)}{\varphi(t)}
$$

are known as coefficients of almost increase (almost decrease, respectively).

The lemmas in this section present a usual tool used in quasi-metric measure spaces under the lower or upper Ahlfors conditions, see, for instance, [16], [22, Lemmas 2.2, 2.4, 2.5 and 2.8], where such statements may be found in a more general form, but either for bounded sets $X$ or unbounded with $\sigma_{0}=\sigma_{\infty}$ and $\tau_{0}=\tau_{\infty}$. However we give their short proof in our case, since we admit $\sigma_{0} \neq \sigma_{\infty}$ and $\tau_{0} \neq \tau_{\infty}$. Lemmas 2.1 - 2.3 may be regarded as an analogue of passage to polar coordinates used in the Euclidean case.

Lemma 2.1. Let $\psi:(0, \ell) \rightarrow(0, \ell)$ be doubling and almost increasing and $a \in \mathbb{R}$.

I. Let $\ell<\infty$. If the triplet $(X, d, \mu)$ satisfes the upper Ahlfors condition (2.6) at the point $x=x_{0}$, then 


$$
\int_{B\left(x_{0}, r\right)} \frac{\psi\left[d\left(y, x_{0}\right)\right]}{d\left(y, x_{0}\right)^{a}} d \mu(y) \leq C \int_{0}^{r} t^{\sigma_{0}-a-1} \psi(t) d t, 0<r<\ell .
$$

If the triplet $(X, d, \mu)$ satisfies the lower Ahlfors condition (2.3) and the condition (2.2) at the point $x_{0}$, then

$$
\int_{0}^{r} t^{\tau_{0}-a-1} \psi(t) d t \leq C \int_{B\left(x_{0}, r\right)} \frac{\psi\left[d\left(y, x_{0}\right)\right]}{d\left(y, x_{0}\right)^{a}} d \mu(y), 0<r<\ell .
$$

II. Let $\ell=\infty$. If $(X, d, \mu)$ satisfies the upper Ahlfors conditions (2.7) and (2.8) at the point $x=x_{0}$, then

$$
\int_{B\left(x_{0}, r\right)} \frac{\psi\left[d\left(y, x_{0}\right)\right]}{d\left(y, x_{0}\right)^{a}} d \mu(y) \leq C \int_{0}^{r} t^{\sigma(t)-a-1} \psi(t) d t, 0<r<\infty,
$$

where $\sigma(t)=\left\{\begin{array}{cc}\sigma_{0}, & 0<t<1 \\ \sigma_{\infty}, & 1<t<\infty .\end{array}\right.$ If $(X, d, \mu)$ satisfies the lower Ahlfors conditions (2.4) and (2.5) and the condition (2.2) at the point $x=x_{0}$, then

$$
\int_{0}^{r} t^{\tau(t)-a-1} \psi(t) d t \leq C \int_{B\left(x_{0}, r\right)} \frac{\psi\left[d\left(y, x_{0}\right)\right]}{d\left(y, x_{0}\right)^{a}} d \mu(y), 0<r<\ell,
$$

where $\tau(t)=\left\{\begin{array}{cc}\tau_{0}, & 0<t<1 \\ \tau_{\infty}, & 1<t<\infty\end{array}\right.$.

P r o o f. I. We have

$$
\begin{gathered}
\int_{B\left(x_{0}, r\right)} \frac{\psi\left[d\left(y, x_{0}\right)\right]}{d\left(y, x_{0}\right)^{a}} d \mu(y)=\sum_{k=-\infty_{2^{k}}<d\left(y, x_{0}\right)<2^{k+1} r}^{k=-1} \frac{\psi\left[d\left(y, x_{0}\right)\right]}{d\left(y, x_{0}\right)^{a}} d \mu(y) \\
\leq C \sum_{k=-\infty}^{k=-1} \psi\left(2^{k+1} r\right) \int_{2^{k} r<d\left(y, x_{0}\right)<2^{k+1} r} d\left(y, x_{0}\right)^{-a} d \mu(y) \\
\leq C \sum_{k=-\infty}^{k=-1} \frac{\psi\left(2^{k+1} r\right)}{\left(2^{k+1} r\right)^{a}} \mu B\left(x_{0}, 2^{k+1} r\right) \\
\leq C \sum_{k=-\infty}^{k=-1} \psi\left(2^{k} r\right)\left(2^{k} r\right)^{\sigma_{0}-a} \leq C \sum_{k=-\infty}^{k=-1}\left(2^{k} r\right)^{\sigma_{0}-a-1} \int_{2^{k} r}^{2^{k+1} r} \psi(t) d t
\end{gathered}
$$




$$
\leq C \sum_{k=-\infty}^{k=-1} \int_{2^{k} r}^{2^{k+1} r} t^{\sigma_{0}-a-1} \psi(t) d t=C \int_{0}^{r} t^{\sigma_{0}-a-1} \psi(t) d t .
$$

The proof of the estimate (2.10) is completed. The inverse estimate (2.11) is analogously proved via diadic decompositions with the use of the lower Ahlfors condition and condition (2.2) taken into account.

II. The proof for unbounded sets $X$ follows the same lines as above, so that we sketch only the principal differences:

We have

$$
\begin{gathered}
\int_{B\left(x_{0}, r\right)} \frac{\psi\left[d\left(y, x_{0}\right)\right]}{d\left(y, x_{0}\right)^{a}} d \mu(y)=\sum_{\substack{k \leq-1: \\
2^{k_{r} \leq 1} 2^{k} r<d\left(y, x_{0}\right)<2^{k+1} r}} \frac{\psi\left[d\left(y, x_{0}\right)\right]}{d\left(y, x_{0}\right)^{a}} d \mu(y) \\
+\sum_{\substack{k \leq-1: \\
2^{k} r>1}} \int_{2^{k} r<d\left(y, x_{0}\right)<2^{k+1} r} \frac{\psi\left[d\left(y, x_{0}\right)\right]}{d\left(y, x_{0}\right)^{a}} d \mu(y) \\
\leq C\left(\sum_{\substack{k \leq-1: \\
2^{k} r \leq 1}} \frac{\psi\left(2^{k} r\right)}{\left(2^{k} r\right)^{a}}\left(2^{k} r\right)^{\sigma_{0}}+\sum_{\substack{k \leq-1: \\
2^{k} r>1}} \frac{\psi\left(2^{k} r\right)}{\left(2^{k} r\right)^{a}}\left(2^{k} r\right)^{\sigma_{\infty}}\right),
\end{gathered}
$$

which yields (2.12) after easy estimations similar to those in the proof of (2.10).

Changes in the proof of (2.11) necessary for the proof of (2.13) are similarly traced.

In a similar way the following lemma is proved.

Lemma 2.2. Let $\ell=\infty$ and $\psi$ satisfy the assumptions of Lemma 2.1 and $b \in \mathbb{R}$. If $(X, d, \mu)$ satisfies the upper Ahlfors conditions (2.7) and (2.8) at the point $x_{0}$, then

$$
\int_{X \backslash B\left(x_{0}, r\right)} \frac{\psi\left[d\left(y, x_{0}\right)\right]}{d\left(y, x_{0}\right)^{b}} d \mu(y) \leq C \int_{r}^{\infty} t^{\sigma(t)-b-1} \psi(t) d t, 0<r<\ell .
$$

If $(X, d, \mu)$ satisfies the lower Ahlfors conditions (2.4), (2.5) and the condition (2.2) at the point $x_{0}$, then

$$
\int_{r}^{\infty} t^{\tau(t)-b-1} \psi(t) d t \leq C \int_{X \backslash B\left(x_{0}, r\right)} \frac{\psi\left[d\left(y, x_{0}\right)\right]}{d\left(y, x_{0}\right)^{b}} d \mu(y), 0<r<\ell .
$$

LEMma 2.3. Let $g$ be a non-negative function on $\mathbb{R}_{+}$, satisfying the doubling condition $g(2 r) \leq c g(r)$ and such that there exists $\omega \geq 0$ such 
that $r^{\omega} g(r)$ is almost increasing. If $(X, d, \mu)$ satisfies the growth conditions (2.7) and (2.8), then

$$
C_{1} \int_{B\left(x_{0}, r\right) \backslash B\left(x_{0}, \frac{r}{2}\right)} g\left[d\left(y, x_{0}\right)\right] d \mu(y) \leq r^{\sigma(r)} g(r) \leq C_{2} \int_{\frac{r}{2}}^{r} t^{\sigma(t)-1} g(t) d t .
$$

P r o o f. With the notation $g_{\omega}(t)=t^{\omega} g(t)$ we have

$$
\begin{gathered}
\int_{B\left(x_{0}, r\right) \backslash B\left(x_{0}, \frac{r}{2}\right)} g\left[d\left(y, x_{0}\right)\right] d \mu(y)=\int_{B\left(x_{0}, r\right) \backslash B\left(x_{0}, \frac{r}{2}\right)} \frac{g_{\omega}\left[d\left(y, x_{0}\right)\right]}{d\left(y, x_{0}\right)^{\omega}} d \mu(y) \\
\leq C \frac{g_{\omega}(r)}{(r / 2)^{\omega}} \mu B(x, r) \leq C r^{\sigma(r)} g(r) .
\end{gathered}
$$

On the other hand,

$$
\int_{r / 2}^{r} t^{\sigma(t)-1} g(t) d t=\int_{r / 2}^{r} t^{\sigma(t)-\omega-1} g_{\omega}(t) d t \geq C g_{\omega}\left(\frac{r}{2}\right) r^{\sigma(r)-\omega} \geq C r^{\sigma(r)} g(r) .
$$

We also need the following lemma which is proved by standard arguments via the diadic decomposition.

Lemma 2.4. Let $\psi:(0, \ell) \rightarrow(0, \ell), 0<\ell \leq \infty$. If $\psi$ is almost decreasing on $(R, \ell)$ for some $0<R<\ell$, then

$$
\sum_{k \geq 0: 2^{k+1} r \leq \ell} \psi\left(2^{k+1} r\right) \leq \frac{c}{\ln 2} \int_{r}^{\ell} \frac{\psi(t)}{t} d t, \quad R<r<\ell
$$

where $c=\sup _{R<r<\varrho} \frac{\psi(\varrho)}{\psi(r)}$. If $\psi$ is almost increasing on $(0, R)$ for some $0<R \leq \ell$, then

$$
\sum_{k=-\infty}^{0} \psi\left(2^{k} r\right) \leq \frac{c}{\ln 2} \int_{0}^{r} \frac{\psi(t)}{t} d t, \quad 0<r<R
$$

where $c=\sup _{0<\varrho<r<R} \frac{\psi(\varrho)}{\psi(r)}$.

\subsection{Generalized Morrey spaces on $(X, d, \mu)$.}

Definition 2.3. By $\mathfrak{F}=\mathfrak{F}([0, \ell))$ we denote the class of non-negative almost increasing measurable functions $\varphi$ on $[0, \ell)$, which satisfy the conditions: 


$$
\inf _{\delta<r<\ell} \varphi(r)>0
$$

for every $\delta>0$.

In next Lemma 2.5 we shell use the condition

$$
\int_{0}^{r} \frac{\varphi(t)}{t} d t \leq C \varphi(r), \quad 0<r<\infty
$$

known as the Zygmund condition, and the condition that there exists an $\varepsilon>0$ such that

$$
\frac{\varphi(r)}{r^{\sigma(r)-\varepsilon}} \text { is almost decreasing, }
$$

where $\sigma(r)$ was introduced in Lemma 2.1.

Let $1 \leq p<\infty$. We will use the notation

$$
\mathfrak{M}_{p, \varphi}(f ; x, r):=\frac{1}{\varphi(r)} \int_{B(x, r)}|f(y)|^{p} d \mu(y) .
$$

We introduce the generalized Morrey spaces, global and local, by the following definition.

Everywhere in the sequel it is supposed that $\varphi \in \mathfrak{F}([0, \ell))$.

Definition 2.4. The generalized Morrey spaces, the global $\mathcal{L}^{p, \varphi}(X)$ and local $\mathcal{L}_{\left\{x_{0}\right\}}^{p, \varphi}(X)$, are defined as the spaces of functions $f \in L_{\text {loc }}^{p}(X)$ having the finite norms

$$
\|f\|_{\mathcal{L}^{p, \varphi}(X)}:=\sup _{r \in(0, \ell)} \sup _{x \in X} \mathfrak{M}_{p, \varphi}(f ; x, r)^{\frac{1}{p}}
$$

and

$$
\|f\|_{\mathcal{L}_{\left\{x_{0}\right\}}^{p, \varphi}(X)}:=\sup _{r \in(0, \ell)} \mathfrak{M}_{p, \varphi}\left(f ; x_{0}, r\right)^{\frac{1}{p}}, \quad x_{0} \in X
$$

respectively.

The spaces $\mathcal{L}^{p, \varphi}(X), \mathcal{L}_{\left\{x_{0}\right\}}^{p, \varphi}(X)$ are Banach function spaces in the sense of [2].

The following lemma provides conditions for the non-triviality of Morrey spaces. We consider the most interesting case $\ell=\infty$. More precisely, it contains sufficient conditions on the function $\varphi$ and the upper Ahlfors 
dimensions $\sigma_{0}$ and $\sigma_{\infty}$ for inclusion of the function

$$
f_{0}(x):=\left(\frac{\varphi\left[d\left(x_{0}, x\right)\right]}{d\left(x_{0}, x\right)^{\sigma\left[d\left(x_{0}, x\right)\right]}}\right)^{\frac{1}{p}}
$$

into local or global Morrey space. In particular, in the case

$\varphi(r)= \begin{cases}r^{\lambda_{0}}, & 0<r<1, \quad \lambda_{0}>0, \quad \lambda_{\infty}>0, \text { corresponding to Morrey } \\ r^{\lambda_{\infty}}, & r>1,\end{cases}$ spaces of classical type, Lemma (2.5) states that the local Morrey space is non-trivial for all $\lambda_{0}>0$ and $\lambda_{\infty}>0$, and global Morrey space is non-trivial for $0<\lambda_{0}<\sigma_{0}$ and $0<\lambda_{\infty}<\sigma_{\infty}$.

Lemma 2.5. Let $1 \leq p<\infty$. Let $\ell=\infty$. Let $\varphi \in \mathfrak{F}$ and $x_{0} \in X$.

Let $(X, d, \mu)$ satisfy the conditions (2.7) and (2.8) only at the point $x=x_{0}$. Then the condition (2.20) is sufficient for the inclusion $f_{0}(x) \in$ $\mathcal{L}_{\left\{x_{0}\right\}}^{p, \varphi}(X)$.

Let $(X, d, \mu)$ satisfy the uniform conditions (2.7) and (2.8). Then the conditions (2.20) and (2.21) are sufficient for the inclusion $f_{0}(x) \in \mathcal{L}^{p, \varphi}(X)$.

P r o o f. For the modular $\mathfrak{M}_{p, \varphi}\left(f_{0} ; x_{0}, r\right)$ we have

$$
\mathfrak{M}_{p, \varphi}\left(f_{0} ; x_{0}, r\right)=\frac{1}{\varphi(r)} \int_{B\left(x_{0}, r\right)} \frac{\varphi\left[d\left(x_{0}, y\right)\right]}{d\left(x_{0}, y\right)^{\sigma\left[d\left(x_{0}, y\right)\right]}} d \mu(y) .
$$

We apply Lemma 2.1 separately considering the cases $r \leq 1$ and $r>1$ and splitting integration to $0<d\left(y, x_{0}\right)<1$ and $1<d\left(y, x_{0}\right)<r$ when $r>1$, and after direct technical steps we obtain

$$
\mathfrak{M}_{p, \varphi}\left(f_{0} ; x_{0}, r\right) \leq \frac{C}{\varphi(r)} \int_{0}^{r} \frac{\varphi(t)}{t} d t .
$$

It remains to apply the Zygmund condition (2.20).

To estimate the modular $\mathfrak{M}_{p, \varphi}\left(f_{0} ; x, r\right)$, we distinguish the cases $d\left(x, x_{0}\right) \leq$ $2 k r$ and $d\left(x, x_{0}\right)>2 k r$, where $k$ is the constant from the triangle inequality. In the first case for $y \in B(x, r)$ by the triangle inequality we have $d\left(y, x_{0}\right) \leq k\left[d\left(x, x_{0}\right)+d(x, y)\right] \leq k_{1} r$, where $k_{1}=k(2 k+1)$, so that $B(x, r) \subset B\left(x_{0}, k_{1} r\right)$, then as in the proof of (2.26) we obtain that

$$
\mathfrak{M}_{p, \varphi}\left(f_{0} ; x, r\right) \leq \frac{C}{\varphi(r)} \int_{0}^{k_{1} r} \frac{\varphi(t)}{t} d t
$$

whence the estimate follows by the Zygmund and doubling conditions for $\varphi$. 
For the case $d\left(x, x_{0}\right)>2 k r$ we represent the modular as

$$
\mathfrak{M}_{p, \varphi}\left(f_{0} ; x, r\right)=\frac{1}{\varphi(r)} \int_{B(x, r)} \frac{\varphi\left[d\left(x_{0}, y\right)\right] d\left(x_{0}, y\right)^{-\varepsilon}}{d\left(x_{0}, y\right)^{\sigma\left[d\left(x_{0}, y\right)\right]-\varepsilon}} d \mu(y)
$$

For $y \in B(x, r)$, by the triangle inequality we have $d\left(y, x_{0}\right) \geq \frac{1}{k} d\left(x, x_{0}\right)+$ $d(x, y)] \geq r$, and then by (2.21)

$$
\mathfrak{M}_{p, \varphi}\left(f_{0} ; x, r\right) \leq \frac{C}{r^{\sigma(r)-\varepsilon}} \int_{B(x, r)} \frac{d y}{d(y, x)^{\varepsilon}} .
$$

It remains to apply Lemma 2.1 with separating the cases $r<1$ and $r>1$ as above.

Given a weight $w$ on $(X, d, \mu)$, we define weighted generalized Morrey spaces in the usual way:

$$
\mathcal{L}^{p, \varphi}(X, w):=\left\{f: w f \in \mathcal{L}^{p, \varphi}(X)\right\} .
$$

The local space $\mathcal{L}_{\left\{x_{0}\right\}}^{p, \varphi}(X, w)$ is similarly defined.

\section{Main results}

Recall that our goal is to obtain sufficient and/or necessary conditions for the embedding

$$
\mathcal{L}_{\left\{x_{0}\right\}}^{p, \varphi}(X, w) \hookrightarrow L^{s}(X), \quad x_{0} \in X, 1 \leq s \leq p<\infty .
$$

3.1. General weights. In Theorem 3.1 we use the notation:

$$
\Psi_{\varphi, w ; x_{0}}(t):=\left\|\frac{\varphi\left[d\left(\cdot, x_{0}\right)\right]^{\frac{1}{p}}}{w(\cdot)}\right\|_{L^{\frac{s p}{p-s}}\left(B\left(x_{0}, t\right) \backslash B\left(x_{0}, \frac{t}{2}\right)\right)}^{s}, 1 \leq s \leq p
$$

where $0<t<\ell$.

We also denote by $\mathcal{G}(0, \ell)$ the class of non-negative functions $\psi(t), t \in$ $(0, \ell)$ satisfying the condition (2.19), such that:

1) $\psi$ is almost increasing on $(0, \ell)$ when $\ell<\infty$, and $\psi$ is almost increasing on $\left(0, t_{0}\right)$ and almost decreasing on $\left(t_{0}, \infty\right)$ for some $t_{0}=t_{0}(\psi) \in$ $\mathbb{R}+$, when $\ell=\infty$;

2) $\int_{0}^{\ell} \frac{\psi(t)}{t} d t<\infty$

REMARK 3.1. Note that if a function $\psi \in \mathcal{G}(0, \infty)$ is bounded on $(0, \infty)$, then the condition 1$)$ in the definition of the class $\mathcal{G}$ holds for any $t_{0}>0$. 
For a function $\psi \in \mathcal{G}$ we denote

$$
k(\psi)= \begin{cases}C_{\psi}(0, \ell), & \text { if } \ell<\infty, \\ \max \left\{C_{\psi}\left(0, t_{0}\right), c_{\psi}\left(t_{0}, \infty\right),\right\}, & \text { if } \ell=\infty,\end{cases}
$$

where the constants $C_{\psi}$ and $c_{\psi}$ were fefined in (2.9).

In Theorem 3.1 we do not suppose that the measure $\mu$ either is doubling or satisfies the growth condition.

Theorem 3.1. Let $1 \leq p<\infty$. Let $\varphi \in \mathfrak{F}([0, \ell))$ and $(X, d, \mu) \in \mathfrak{X}$. Let $w$ be a weight on $(X, d, \mu)$. If $\Psi_{\varphi, w ; x_{0}} \in \mathcal{G}$, then the embedding (3.1) holds and

where

$$
\|f\|_{L^{s}(X)} \leq\left[C_{\varphi} \cdot C\left(x_{0}\right)\right]^{\frac{1}{s}}\|f\|_{L_{\left\{x_{0}\right\}}^{p, \varphi}(X, w)},
$$

$$
C\left(x_{0}\right)=\left(\frac{k\left(\Psi_{\varphi, w ; x_{0}}\right)}{\ln 2} \int_{0}^{\ell} \frac{\Psi_{\varphi, w ; x_{0}}(t)}{t} d t\right)
$$

and $C_{\varphi}$ was defined in (2.9).

P r o o f. We use the diadic decomposition and obtain

$$
\int_{X}|f(y)|^{s} d \mu(y)=\sum_{k=-\infty_{B_{k}}(r)}^{k=\infty} \int_{\varphi\left(\left[d\left(y, x_{0}\right)\right]^{\frac{1}{p}}\right.}\left|\frac{f(y) w(y)}{\varphi}\right|^{s}\left[\frac{\varphi\left[d\left(y, x_{0}\right)\right]^{\frac{1}{p}}}{w(y)}\right]^{s} d \mu(y),
$$

where $B_{k}(r)=B\left(x_{0}, 2^{k+1} r\right) \backslash B\left(x_{0}, 2^{k} r\right)$ and $r>0$ will be chosen later.

Let first $X$ be bounded. We choose $r=\ell$ in (3.6) then all the terms in the sum $\sum_{k=-\infty}^{k=\infty}$ with $k \geq 0$ disappear.

By the Hölder inequality with the exponents $q=\frac{p}{s}$ and $q^{\prime}=\frac{p}{p-s}$ we then have

$$
\int_{X}|f(y)|^{s} d \mu(y) \leq \sum_{k=-\infty}^{k=-1} \Psi_{\varphi, w ; x_{0}}\left(2^{k+1} \ell\right)\left(\int_{B_{k}(\ell)} \frac{|f(y) w(y)|^{p}}{\varphi\left[d\left(y, x_{0}\right)\right]} d \mu(y)\right)^{\frac{s}{p}} .
$$

Since $\varphi$ is almost increasing, we have $\frac{1}{\varphi\left[d\left(y, x_{0}\right)\right]} \leq \frac{C_{\varphi}}{\varphi\left(2^{k} \ell\right)}$ and we obtain

$$
\int_{X}|f(y)|^{s} d \mu(y) \leq C_{\varphi}\|f\|_{\mathcal{L}_{\left\{x_{0}\right\}}^{p, \varphi}(X, w)}^{s} \sum_{k=-\infty}^{k=-1} \Psi_{\varphi, w ; x_{0}}\left(2^{k+1} \ell\right) .
$$

It remains to apply the estimate (2.18) of Lemma 2.4 with $R=\ell$. The choice $R=\ell$ is possible. Indeed if $\Psi_{\varphi, w ; x_{0}}$ is almost increasing on a small 
interval $[0, \delta]$, then by (2.19) it is easy to see that the function $\Psi_{\varphi, w ; x_{0}}$ is almost increasing on the whole interval $(0, \ell)$. We obtain

$$
\|f\|_{L^{s}(X)}^{s} \leq \frac{C\left(x_{0}, \ell\right)}{\ln 2} \int_{0}^{\ell} \frac{\Psi_{\varphi, w ; x_{0}}(t)}{t} d t\|f\|_{\mathcal{L}_{\left\{x_{0}\right\}}^{p, \varphi}(X, w)}^{s} .
$$

Let now $X$ be unbounded. In (3.6) we choose $r=t_{0}$, where $t_{0}=$ $t_{0}\left(\Psi_{\varphi, w ; x_{0}}\right)$ is the point from the definition of the class $\mathcal{G}$.

Repeating the same arguments as above, but now with both positive and negative $k$ in the sum $\sum_{k=-\infty}^{k=\infty}$, we obtain

$$
\int_{X}|f(y)|^{s} d \mu(y) \leq\|f\|_{\mathcal{L}_{\left\{x_{0}\right\}}^{p, \varphi}(X, w)}^{s} \sum_{k=-\infty}^{k=\infty} \Psi_{\varphi, w ; x_{0}}\left(2^{k+1} t_{0}\right) .
$$

Then, by Lemma 2.4 we have

$$
\int_{X}|f(y)|^{s} d \mu(y) \leq \frac{k\left(\Psi_{\varphi, w ; x_{0}}\right)}{\ln 2} \int_{0}^{\infty} \frac{\Psi_{\varphi, w ; x_{0}}(t)}{t} d t\|f\|_{\mathcal{L}_{\left\{x_{0}\right\}}^{p, \varphi}(X, w)}^{s},
$$

where the constant $k(\cdot)$ is defined in (3.3). This completes the proof.

The integral $\int_{0}^{\ell} \frac{\Psi \varphi, w ; x_{0}(t)}{t} d t$ involved in (3.4), in case of bounded sets may be majorized by a simpler expression as shown in the following lemma, where we take $s<p$ for simplicity.

Lemma 3.1. Let $1 \leq p<\infty$. Let $\ell<\infty, 1 \leq s<p$ and $\eta<1$. Then the estimate

$$
\int_{0}^{\ell} \frac{\Psi_{\varphi, w ; x_{0}}(t)}{t} d t \leq C\left(\int_{X}\left[\frac{\varphi\left[d\left(y, x_{0}\right)\right]^{\frac{1}{p}}}{w(y)}\right]^{\frac{s p}{p-s}} d\left(y, x_{0}\right)^{\eta} d \mu(y)\right)^{1-\frac{s}{p}}
$$

holds, where $C=C(p, s, \eta ; \ell)$.

P r o o f. With $q=\frac{p}{p-s}$ in the case $s<p$ we have

$$
\int_{0}^{\ell} \frac{\Psi_{\varphi, w ; x_{0}}(t)}{t} d t=\int_{0}^{\ell} t^{\frac{1-\eta}{q}-1}\left(t^{\eta-1} \int_{B\left(x_{0}, t\right) \backslash B\left(x_{0}, \frac{t}{2}\right)}\left[\frac{\varphi\left[d\left(y, x_{0}\right)\right]^{\frac{1}{p}}}{w(y)}\right]^{s q} d \mu(y)\right)^{\frac{1}{q}} d t .
$$

Applying the Hölder inequality with the exponent $q$ and interchanging the order of integration, we obtain 


$$
\begin{gathered}
\int_{0}^{\ell} \frac{\Psi_{\varphi, w ; x_{0}}(t)}{t} d t \\
\leq\left(\int_{0}^{\ell} t^{\frac{p}{s}\left(\frac{1-\eta}{q}-1\right)} d t\right)^{\frac{1}{q^{\prime}}}\left(\int_{X}\left[\frac{\varphi\left[d\left(y, x_{0}\right)\right]^{\frac{1}{p}}}{w(y)}\right]^{s q} d \mu(y) \int_{d\left(y, x_{0}\right)}^{2 d\left(y, x_{0}\right)} t^{\eta-1} d t\right)^{\frac{1}{q}}
\end{gathered}
$$

and we arrive at (3.9) after easy calculations.

Corollary 3.1. Let the assumptions of Theorem 3.1 hold for some fixed point $x_{0} \in X$. Then the same embedding holds for the global space:

holds.

$$
\mathcal{L}^{p, \varphi}(X, w) \hookrightarrow L^{s}(X), \quad 1 \leq s \leq p,
$$

P r o o f. It suffices to apply (3.4) and use the fact that $\|f\|_{L_{\left\{x_{0}\right\}}^{p, \varphi}(X, w)} \leq$ $\|f\|_{L^{p, \varphi}(X, w)}$

3.2. Radial weights: sufficient conditions. We call a weight radial if it depends on a distance $d\left(y, y_{0}\right)$ to some point $y_{0} \in X$. Below we choose $y_{0}$ as the center $x_{0}$ of our Morrey space: $y_{0}=x_{0}$, which is natural for the study of local Morrey spaces.

In Theorem 3.2 we deal with radial weights $w=w\left[d\left(y, x_{0}\right)\right]$ and we consider the case diam $X=\infty$, which is of main interest.

Instead of the function defined in (3.2) we consider its modification

$\Phi_{\varphi, w}^{\sigma}(t):=\left\{\begin{array}{l}\left(\int_{t / 2}^{t} \varrho^{\sigma(\varrho)-1}\left[\frac{\varphi(\varrho)^{\frac{1}{p}}}{w(\varrho)}\right]^{\frac{s p}{p-s}} d \varrho\right)^{\frac{p-s}{p}}, \quad \text { if } 1 \leq s<p, \quad 0<t<\infty, \\ \sup _{t / 2<\varrho<t} \frac{\varphi(\varrho)^{\frac{1}{p}}}{w(\varrho)}, \quad \text { if } s=p,\end{array}\right.$

which is in fact the dominant of $\Psi_{\varphi, w ; x_{0}}$ in view of Lemma 2.3 .

Theorem 3.2. Let $1 \leq p<\infty$. Let $\varphi \in \mathfrak{F}([0, \ell))$ and let $(X, d, \mu) \in \mathfrak{X}$ satisfy the growth condition (2.6). Then the embedding 3.1 holds under the assumption that:

i) the function $u(t):=\frac{\varphi(t)}{w(t)^{p}}$ is doubling: $u(2 t) \leq c u(t), t \in \mathbb{R}_{+}$, and has the property that there exists an $\omega>0$ such that $t^{\omega} u(t)$ is almost increasing on $\mathbb{R}_{+}$;

and 
ii) $\Phi_{\varphi, w}^{\sigma} \in \mathcal{G}(0, \ell)$.

P r o o f. For the function (3.2) we have $\Psi_{\varphi, w ; x_{0}} \leq C \Phi_{\varphi, w}^{\sigma}(t)$, which under our assumptions follows from Lemma 2.3 with $g(r)=u(r)^{\frac{1}{p}}$. Thus, this theorem formally follows from Theorem [3.1. However, since the assumptions in this theorem do not guarantee in general that $\Psi_{\varphi, w ; x_{0}} \in \mathcal{G}$, we make an independent proof and start as in the proof of Theorem 3.1 via diadic decomposition:

$$
\int_{X}|f(y)|^{s} d \mu(y)=\sum_{k=-\infty_{B_{k}(r)}}^{k=\infty}\left|\frac{f(y) w\left[d\left(y, x_{0}\right)\right]}{\varphi\left(\left[d\left(y, x_{0}\right)\right]^{\frac{1}{p}}\right.}\right|^{s}\left(\frac{\varphi\left[d\left(y, x_{0}\right)\right]^{\frac{1}{p}}}{w\left[d\left(y, x_{0}\right)\right]}\right)^{s} d \mu(y) .
$$

By the Hölder inequality with $q=\frac{p}{s}$ and $q^{\prime}=\frac{p}{p-s}$ we obtain

$$
\begin{gathered}
\int_{X}|f(y)|^{s} d \mu(y) \leq \sum_{k=-\infty}^{k=\infty}\left(\int_{B_{k}(r)}\left(\frac{\varphi\left[d\left(y, x_{0}\right)\right]^{\frac{1}{p}}}{w\left[d\left(y, x_{0}\right)\right]}\right)^{s q^{\prime}} d \mu(y)\right)^{\frac{1}{q^{\prime}}} \\
\times\left(\int_{B_{k}\left(x_{0}, 2^{k+1} r\right)}\left|\frac{f(y) w\left[d\left(y, x_{0}\right)\right]}{\varphi\left(\left[d\left(y, x_{0}\right)\right]^{\frac{1}{p}}\right.}\right|^{p}\right)^{\frac{1}{q}} d \mu(y) \\
\leq\|f\|_{\mathcal{L}_{\left\{x_{0}\right\}}^{p, \varphi}(X, w)} \sum_{k=-\infty}^{k=\infty}\left(\int_{B_{k}(r)}\left(\frac{\varphi\left[d\left(y, x_{0}\right)\right]^{\frac{1}{p}}}{w\left[d\left(y, x_{0}\right)\right]}\right)^{s q^{\prime}} d \mu(y)\right)^{\frac{1}{q^{\prime}}} .
\end{gathered}
$$

By Lemma 2.3 we obtain

$$
\begin{gathered}
\int_{X}|f(y)|^{s} d \mu(y) \leq C\|f\|_{\mathcal{L}_{\left\{x_{0}\right\}}^{p, \varphi}(X, w)}^{s} \sum_{k=-\infty}^{k=\infty}\left(\int_{2^{k} r}^{2^{k+1} r} t^{\sigma(t)-1}\left(\frac{\varphi(t)^{\frac{1}{p}}}{w(t)}\right)^{\frac{s p}{p-s}} d t\right)^{\frac{p-s}{p}} \\
=C\|f\|_{\mathcal{L}_{\left\{x_{0}\right\}}^{p, \varphi}(X, w)}^{s=\infty} \sum_{k=-\infty}^{k=\infty} \Phi_{\varphi, w}\left(2^{k+1} r\right) .
\end{gathered}
$$

Now we choose $r=t_{0}=t_{0}\left(\Phi_{\varphi, w}\right)$, where $t_{0}$ is the point in $\mathbb{R}_{+}$from the definition of the class $\mathcal{G}$. Then Lemma 2.4 is applicable which yields

$$
\int_{X}|f(y)|^{s} d \mu(y) \leq C \int_{0}^{\infty} \frac{\Phi_{\varphi, w}(t)}{t} d t\|f\|_{\mathcal{L}^{p, \varphi}(X, w)}^{s} .
$$

The proof is completed. 
In the next theorem we consider the case of power-logarithmic-type radial weights

where $\alpha, \beta, \gamma, \delta \in \mathbb{R}$.

$$
w(r)=\left\{\begin{array}{ll}
r^{\alpha}\left(\ln \frac{e}{r}\right)^{\gamma}, & r<1, \\
r^{\beta}(\ln e r)^{\delta}, & r>1,
\end{array}, \quad r=d\left(y, x_{0}\right),\right.
$$

Theorem 3.3. Let $1 \leq p<\infty$. Let $\varphi \in \mathfrak{F}([0, \ell))$ and $\varphi(2 t) \leq c \varphi(t)$, and let $w$ be the weight (3.13). Then the embedding (3.1) with the weight $w=w\left[d\left(x, x_{0}\right)\right]$ holds if

$$
\begin{gathered}
\varphi(t) t^{\sigma_{0} \frac{p-s}{s}-\alpha p}\left(\ln \frac{e}{t}\right)^{-\gamma p} \text { is almost increasing on }(0,1) \text { and } \\
\varphi(t) t^{\sigma_{\infty} \frac{p-s}{s}-\beta p}(\ln e t)^{-\delta p} \text { is almost decreasing on }(1, \infty)
\end{gathered}
$$

and

$$
\begin{gathered}
\int_{0}^{1} \varphi(t)^{\frac{s}{p}} t^{\sigma_{0} \frac{p-s}{p}-\alpha s-1}\left(\ln \frac{e}{t}\right)^{-\gamma s} d t<\infty \text { and } \\
\int_{1}^{\infty} \varphi(t)^{\frac{s}{p}} t^{\sigma_{\infty} \frac{p-s}{p}-\beta s-1}(\ln (e t))^{-\delta s} d t<\infty .
\end{gathered}
$$

P r o o f. The proof reduces to the straightforward verification of conditions of Theorem 3.2. Indeed, the case $s=p$ being simple, we consider the case $s<p$. With the weight (3.13), for $0<t<1$ we have

$$
\Phi_{\varphi, w}^{\sigma}(t)=\left(\int_{t / 2}^{t} \varphi(\varrho)^{\frac{s}{p-s}} \varrho^{\sigma_{0}-\alpha q-1}\left(\ln \frac{e}{\varrho}\right)^{-\gamma q} d \varrho\right)^{\frac{s}{q}}, \quad q=\frac{p s}{p-s} .
$$

Since $\varphi$ is almost increasing, after the delation change of variables, we obtain

$$
\Phi_{\varphi, w}^{\sigma}(t) \leq C_{\varphi}^{\frac{s}{p}} \varphi(t)^{\frac{s}{p}} t^{\left(\frac{\sigma_{0}}{q}-\alpha\right) s}\left(\int_{1 / 2}^{1} \xi^{\sigma_{0}-1-\alpha q}\left[\ln \frac{\sqrt{e}}{t}+\ln \frac{\sqrt{e}}{\xi}\right]^{-\gamma q} d \xi\right)^{\frac{s}{q}} .
$$

Similarly for $t>2$ we have $\Phi_{\varphi, w}^{\sigma}(t)$,

$$
\leq C_{\varphi}^{\frac{s}{p}}\left|S^{\sigma_{\infty}-1}\right|^{\frac{1}{q}} \varphi(t)^{\frac{s}{p}} t\left(\frac{\sigma_{\infty}}{q}-\beta\right) s\left(\int_{1 / 2}^{1} \xi^{\sigma_{\infty}-1-\beta q}\left[\ln \frac{e}{2} t+\ln (2 \xi)\right]^{-\delta q} d \xi\right)^{\frac{s}{q}} .
$$

Hence, 


$$
\Phi_{\varphi, w}^{\sigma}(t) \cong\left\{\begin{array}{l}
\varphi(t)^{\frac{s}{p}} t^{\frac{\sigma_{0}}{p}(p-s)-\alpha s}\left(\ln \frac{e}{t}\right)^{-\gamma s}, t<1 \\
\varphi(t)^{\frac{s}{p}} t^{\frac{\sigma_{\infty}}{p}(p-s)-\beta s}(\ln e t)^{-\delta s}, t>1
\end{array}\right.
$$

Corollary 3.2. In the case of Morrey spaces of classical type with $\varphi(r)= \begin{cases}r^{\lambda_{0}}, & 0<r<1, \quad \lambda_{0}>0, \lambda_{\infty}>0, \text { the embedding (3.1) holds, } \\ r^{\lambda_{\infty}}, & r>1,\end{cases}$ if

$\alpha<\frac{\lambda_{0}}{p}+\sigma_{0}\left(\frac{1}{s}-\frac{1}{p}\right)$ and $\gamma \in \mathbb{R}$ or $\alpha=\frac{\lambda_{0}}{p}+\sigma_{0}\left(\frac{1}{s}-\frac{1}{p}\right)$ and $\gamma>\frac{1}{s}$,

$\beta>\frac{\lambda_{\infty}}{p}+\sigma_{\infty}\left(\frac{1}{s}-\frac{1}{p}\right)$ and $\delta \in \mathbb{R}$ or $\beta=\frac{\lambda_{\infty}}{p}+\sigma_{\infty}\left(\frac{1}{s}-\frac{1}{p}\right)$ and $\delta>\frac{1}{s}$.

In the next corollary, taking into account that $(X, d, \mu)$ in general has fractal nature, we specially reformulate the conditions for the embedding (3.1) with the weight (3.13) in terms of restrictions on the upper dimension of fractal sets, arising in the case $s<p$. For simplicity we take $\sigma_{0}=\sigma_{\infty}$.

Corollary 3.3. Let $\sigma_{0}=\sigma_{\infty}:=\sigma$ and $\varphi(r)= \begin{cases}r^{\lambda_{0}}, & 0<r<1, \\ r^{\lambda_{\infty}}, & r>1,\end{cases}$ $\lambda_{0}>0, \lambda_{\infty}>0$, and $w$ be the weight (3.13). Given $p \in(1, \infty), s \in[1, p)$ and the parameters of the weight $w$ satisfying the conditions

$$
\beta>\frac{\lambda_{\infty}}{p} \text { and } \alpha-\beta \leq \frac{\lambda_{0}-\lambda_{\infty}}{p},
$$

the embedding (3.1) holds in the following cases, where we denote $q=$ $\frac{p s}{p-s}$ :

1) $\max \left\{0,\left(\alpha-\frac{\lambda_{0}}{p}\right) q\right\}<\sigma<\left(\beta-\frac{\lambda_{\infty}}{p}\right) q, \alpha-\beta<\frac{\lambda_{0}-\lambda_{\infty}}{p}, \gamma, \delta \in \mathbb{R}$,

2) $\sigma=\left(\alpha-\frac{\lambda_{0}}{p}\right) q$ in the case $\alpha>\frac{\lambda_{0}}{p}, \alpha-\beta<\frac{\lambda_{0}-\lambda_{\infty}}{p}, \gamma>\frac{1}{s}, \delta \in \mathbb{R}$, or $\sigma=\left(\beta-\frac{\lambda_{\infty}}{p}\right) q, \alpha-\beta<\frac{\lambda_{0}-\lambda_{\infty}}{p}$ and $\gamma \in \mathbb{R}, \delta>\frac{1}{s}$,

3) $\sigma=\left(\alpha-\frac{\lambda_{0}}{p}\right) q, \quad \alpha-\beta=\frac{\lambda_{0}-\lambda_{\infty}}{p}$ and $\gamma>\frac{1}{s}, \delta>\frac{1}{s}$ (the unique choice for the upper Ahlfors dimension of $X$ !)

The conditions for embeddings obtained in this subsection are sufficient. Necessary conditions are considered in the next subsection. 
3.3. Radial weights: necessary conditions and criteria for powerlogarithmic weights. Let $w$ be a radial weight. As in subsection 3.2 here we suppose that diam $X=\infty$. In this subsection we suppose that $(X, d, \mu)$ satisfy both the lower and upper Ahlfors conditions (2.4), (2.5), (2.7) and (2.8) at the point $x_{0}$, with the exponents $\tau_{0}, \tau_{\infty}, \sigma_{0}, \sigma_{\infty}$. Note that $\sigma_{0} \leq \tau_{0}$ and $\tau_{\infty} \leq \sigma_{\infty}$.

Theorem 3.4. Let the triplet $(X, d, \mu)$ satisfy the lower and upper Ahlfors conditions (2.4), (2.5), (2.7) and (2.8) at the point $x=x_{0}$. Let $1<p<\infty, 1 \leq s \leq p, \varphi \in \mathfrak{F}\left(\mathbb{R}_{+}\right)$, and the assumption i) of Theorem 3.2 be satisfied.

If the Zygmund condition (2.20) holds, then the condition

$$
\int_{0}^{\infty} t^{\tau(t)-\frac{\sigma(t) s}{p}}\left[\frac{\varphi(t)^{\frac{1}{p}}}{w(t)}\right]^{s} \frac{d t}{t}<\infty
$$

is necessary for the embedding (3.1).

Let the triplet $(X, d, \mu)$ satisfy the uniform lower and upper Ahlfors conditions (2.4), (2.5), (2.7) and (2.8). If additionally the condition (2.21) holds, then the condition (3.18), is necessary also for the embedding (3.10) of the global Morrey space.

P r o o f. Suppose that the embedding (3.1) holds. Introduce the function

$$
f_{w}(x):=\frac{\varphi\left[d\left(x_{0}, x\right)\right]^{\frac{1}{p}}}{w\left[d\left(x_{0}, x\right)\right]\left[d\left(x_{0}, x\right)\right]^{\frac{\sigma\left[d\left(x_{0}, x\right)\right]}{p}}} .
$$

It belongs to $\mathcal{L}_{\left\{x_{0}\right\}}^{p, \varphi}(X, w)$ by Lemma 2.5. Then by the embedding (3.1) we have

$$
\int_{X}\left|f_{w}(x)\right|^{s} d \mu(x) \leq \infty .
$$

By Lemmas 2.1 and 2.2 we obtain that

$$
\int_{X}\left|f_{w}(x)\right|^{s} d \mu(x) \geq C \int_{0}^{\infty} t^{\tau(t)-\frac{\sigma(t) s}{p}}\left[\frac{\varphi(t)^{\frac{1}{p}}}{w(t)}\right]^{s} \frac{d t}{t}
$$

and thus, arrive at the necessity of the condition (3.18).

In the next theorem we consider the case of power-logarithmic-type radial weights (3.13)

THEOREM 3.5. Let the triplet $(X, d, \mu)$ satisfy the lower and upper Ahlfors conditions (2.4), (2.5), (2.7) and (2.8) at the point $x=x_{0}$. Let 
$\varphi \in \mathfrak{F}([0, \ell))$, and let $w$ be the weight (3.13). If $\varphi$ satisfies the Zygmund condition (2.20), then for the embedding (3.1) with the weight $w=w\left[d\left(x, x_{0}\right)\right]$ to hold, the following conditions

$$
\begin{array}{r}
\int_{0}^{1} \varphi(t)^{\frac{s}{p}} t^{\tau_{0}-s\left(\frac{\sigma_{0}}{p}+\alpha\right)-1}\left(\ln \frac{e}{t}\right)^{-\gamma s} d t<\infty \text { and } \\
\int_{1}^{\infty} \varphi(t)^{\frac{s}{p}} t^{\tau_{\infty}-s\left(\frac{\sigma_{\infty}}{p}+\beta\right)-1}(\ln e t)^{-\delta s} d t<\infty .
\end{array}
$$

are necessary.

Let the triplet $(X, d, \mu)$ satisfy the uniform lower and upper Ahlfors conditions (2.4), (2.5), (2.7) and (2.8). The conditions (3.19) are necessary also for the embedding (3.10) of global Morrey space, if additionally (2.21) holds.

P r o o f. It suffices to apply Theorem 3.5 and interpret the condition (3.18) under the choice (3.13) of the weight.

REMARK 3.2. In the case where $(X, d, \mu)$ is regular at the point $x_{0}$, i.e. lower and upper Ahlfors dimensions coincide with each other, both local and global ones: $\tau_{0}=\sigma_{0}$ and $\tau_{\infty}=\sigma_{\infty}$, the necessary condition (3.19) coincides with the condition (3.15), obtained in the sufficiency part for the embedding.

Corollary 3.4. Let $(X, d, \mu)$ be regular at the point $x_{0}$, i.e. lower and upper Ahlfors dimensions coincide with each other, both local and global ones: $\tau_{0}=\sigma_{0}$ and $\tau_{\infty}=\sigma_{\infty}$, and let $\varphi(r)= \begin{cases}r^{\lambda_{0}}, & 0<r<1, \quad \lambda_{0}> \\ r^{\lambda_{\infty}}, & r>1,\end{cases}$ $0, \lambda_{\infty}>0$. Then the embedding (3.1) holds, if and only if $\alpha<\frac{\lambda_{0}}{p}+\sigma_{0}\left(\frac{1}{s}-\frac{1}{p}\right)$ and $\gamma \in \mathbb{R}$ or $\alpha=\frac{\lambda_{0}}{p}+\sigma_{0}\left(\frac{1}{s}-\frac{1}{p}\right)$ and $\gamma>\frac{1}{s}$, $\beta>\frac{\lambda_{\infty}}{p}+\sigma_{\infty}\left(\frac{1}{s}-\frac{1}{p}\right)$ and $\delta \in \mathbb{R}$ or $\beta=\frac{\lambda_{\infty}}{p}+\sigma_{\infty}\left(\frac{1}{s}-\frac{1}{p}\right)$ and $\delta>\frac{1}{s}$.

Let the triplet $(X, d, \mu)$ be regular uniformly for all $x \in X$, and $0<$ $\lambda_{0}<\sigma_{0}, 0<\lambda_{\infty}<\sigma_{\infty}$. Then the embedding (3.10) of global Morrey space holds, if and only if the conditions (3.20)-(3.21) are satisfied. 
3.4. Application to fractal plane curves. In the following example of $(X, d, \mu)$ we consider locally rectifiable plane curves $\Gamma$ of infinite length, which may be represented in the form

$$
\Gamma:=\left\{t \in R^{2}: t=t(\xi), 0<\xi<\infty\right\}
$$

where $\xi$ stands for the arc length. We interpret the distance $d\left(t_{1}, t_{2}\right)$ between $t_{1}, t_{2} \in \Gamma$ as the Euclidean distance on the plane between $t_{1}$ and $t_{2}$, and measure $\mu$ as the arc length measure on $\Gamma$. Morrey spaces on $\Gamma$ are correspondingly interpreted. We assume that $\Gamma$ is a regular, fractal in general, curve in the sense that

$$
c_{1} r^{\sigma} \leq \mu \Gamma\left(t_{0}, r\right) \leq c_{2} r^{\sigma}, t_{0} \in \Gamma, 0<r<\infty,
$$

where $\Gamma\left(t_{0}, r\right)=\left\{t \in \Gamma:\left|t-t_{0}\right|<r\right\}$ and $c_{1}$ and $c_{2}$ do not depend on $t_{0}$ and $r$.

It is clear that $0<\sigma \leq 1$. For curves of fractional dimensions we refer, for instance, to the book [25].

Let $\Gamma$ be an infinite curve satisfying the condition (3.22) and $\varphi$ and $w$ be the same as in Corollary 3.4. The embeddings (3.1) and (3.10) hold if and only if the conditions (3.20)-(3.21) are satisfied.

\section{References}

[1] A. Almeida and S. Samko, Embeddings of local generalized Morrey spaces between weighted Lebesgue spaces. Nonlinear Analysis $\mathbf{1 6 4}$ (2017), 67-76.

[2] C. Bennett and R. Sharpley, Interpolation of operators. Pure and Applied Mathematics 129, Academic Press Inc. Boston, MA (1988).

[3] A. Caetano, Eigenvalue asymptotics of the stokes operator for fractal domains. Proc. London Math. Soc. 76, No 3 (1998), 579-602.

[4] A. Caetano, Growth envelopes of Besov spaces on fractal $h$-sets. Math. Nachr. 286, No 5-6 (2013), 550-568.

[5] A. Caetano and A. Carvalho, On the Hausdorff dimension of continuous functions belonging to Hölder and Besov spaces on fractal $d$-sets. $J$. Fourier Anal. Appl. 18, No 2 (2012), 386-409.

[6] A. Caetano and D. Haroske, Embeddings of Besov spaces on fractal $h$-sets. Banach J. Math. Anal. 9, No 4 (2015), 259-295.

[7] D. Edmunds, V. Kokilashvili, and A. Meskhi, Bounded and Compact Integral Operators. Math. and its Appl. 543, Kluwer Acad. Publ. (2002).

[8] V. Guliyev and S. Samko, Maximal operator in variable exponent generalized Morrey spaces on quasi-metric measure space. Mediterr. J. Math. 13, No 3 (2016), 1151-1165. 
[9] D. Haroske and I. Piotrowska, Atomic decompositions of function spaces with muckenhoupt weights, and some relations to fractal analysis. Math. Nachr. 281, No 10 (2008), 1476-1494.

[10] D. Haroske and L. Skrzypczak, Embeddings of weighted Morrey spaces. Math. Nachr. 290, No 7 (2017), 1066-1086.

[11] J. Heinonen, Lectures on Analysis on Metric Spaces. Universitext, Springer-Verlag, New York (2001).

[12] V. Kokilashvili and A. Meskhi, Fractional integrals on measure spaces. Fract. Calc. Appl. Anal. 4, No 1 (2001), 1-24.

[13] V. Kokilashvili and A. Meskhi, Boundedness of maximal and singular operators in Morrey spaces with variable exponent. Armenian J. Math. 1, No 1 (2008), 18-28.

[14] V. Kokilashvili and A. Meskhi, Maximal and potential operators in variable Morrey spaces defined on nondoubling quasimetric measure spaces. Bull. Georgian Natl. Acad. Sci. (N.S.) 2, No 3 (2008), 18-21.

[15] V. Kokilashvili, A. Meskhi, H. Rafeiro, and S. Samko, Integral Operators in Non-standard Function Spaces, I and II. Springer-Birkhäser (2016).

[16] D. Lukkassen, L.-E. Persson, and N. Samko, Hardy type operators in local vanishing morrey spaces on fractal sets. Fract. Calc. Appl. Anal. 18, No 5 (2015), 1252-1276; DOI: 10.1515/fca-2015-0072; https://www.degruyter.com/view/j/fca.2015.18.issue-5/

issue-files/fca.2015.18.issue-5.xml.

[17] A. Meskhi, H. Rafeiro, and M.A. Zaighum, Interpolation on variable morrey spaces defined on quasi-metric measure spaces. J. Funct. Anal. 270, No 10 (2016), 3946-3961.

[18] E. Nakai, The Campanato, Morrey and Hölder spaces on spaces of homogeneous type. Studia Math. 176, No 1 (2006), 1-19.

[19] H. Rafeiro, N. Samko and S. Samko, Morrey-Campanato Spaces: An Overview. In: Operator Theory: Advances and Applications 228, Birkhäuser, Basel (2013), 293-324.

[20] H. Rafeiro and S. Samko, Fractional integrals and derivatives: Mapping properties. Fract. Calc. Appl. Anal. 19, No 3 (2016), 580-607; DOI: 10.1515/fca-2016-0032; https://www.degruyter.com/view/j/ fca.2016.19.issue-3/issue-files/fca.2016.19.issue-3.xml.

[21] N. Samko, Singular integral operators in weighted spaces with generalized Hölder condition. Proc. A. Razmadze Math. Inst. 120 (1999), 107-134.

[22] N. Samko, S. Samko, and B. Vakulov, Fractional integrals and hypersingular integrals in variable order Hölder spaces on homogeneous spaces. J. Funct. Spaces and Appl. 8, No 3 (2010), 215-244. 
[23] S. Samko, Morrey spaces are closely embedded between vanishing Stummel spaces. Math. Ineq. Appl. 17, No 2 (2014), 627-639.

[24] K. Stempak and X. Tao, Local Morrey and Campanato spaces on quasimetric measure spaces. J. Funct. Spaces 2014 (2014), Article ID 172486, 15 pages.

[25] C. Tricot, Curves and Fractal Dimension. Springer, New York (1994).

[26] H. Triebel, Fractals and Spectra. EMS Tracts in Mathematics, Birkhäuser, Basel (1997).

[27] H. Triebel, A new approach to function spaces on quasi-metric spaces. Rev. Mat. Complut 18, No 1 (2005), 7-48.

[28] H. Triebel, Theory of Function Spaces III. Birkhäuser, Basel (2006).

UiT The Arctic University of Norway

PO Box 385

8505 Narvik, NORWAY

e-mail: Natasha.G.Samko@uit.no

Received: January 25, 2019

Please cite to this paper as published in:

Fract. Calc. Appl. Anal., Vol. 22, No 5 (2019), pp. 1203-1224,

DOI: 10.1515/fca-2019-0064; at https://www.degruyter.com/view/j/fca 